\title{
Model Reference Adaptive Control for DC Motor Based on Simulink
}

\author{
Munadi, M. Amirullah Akbar \\ Department of Mechanical Engineering \\ Diponegoro University \\ Tembalang, Semarang 50275, Indonesia \\ munadi@undip.ac.id,amir240891@gmail.com
}

\author{
Tomohide Naniwa, Yoshiaki Taniai \\ Department of Human and Artificial Intelligent Systems, \\ University of Fukui \\ 3-9-1 Bunkyo, Fukui 910-8507, Japan \\ naniwa@rbt.his.u-fukui.ac.jp, taniai@rbt.his.u-fukui.ac.jp
}

\begin{abstract}
Sometimes the conventional feedback control can not work well to cope with the changes that vary in its dynamic system. The parameters of the dynamic system that changes with time lead to a conventional feedback control system is not able to maintain control. This is caused by circumstances which are nonlinear and receive many disturbance so that the transient response of the system to be less precise and accurate to the desired steady state conditions. To overcome these problems, this paper presents an adaptive control system which can cope with the change of the dynamic DC motor system. The adaptive control system used is Model Reference Adaptive Control (MRAC) which contain four part. They are reference model, adaptation mechanism, plant, and control law. We designs DC motor plant by using Simscape in Simulink. An ideal response for reference model is designed by modeling the dynamic DC motor. Design of an adaptation mechanism with MIT rule and control law with Proportional Integral Derivative (PID) controller are present. It can affect the response of adaptive control system and matching the desired ideal response from reference model even when the system receives a disturbance.
\end{abstract}

Keywords- DC motor; MIT rule; MRAC; PID controller

\section{INTRODUCTION}

A DC motor is the most widely applied actuator in the industry, especially for robotic actuators such as in the control position of an arm robot manipulator [1,2]. DC motors in the industry have a high performance and receive a lot of disturbances caused by the working environment of uncertainty. Some researchers studied the nonlinear condition of DC motors as in $[3,4]$. With these conditions, simple controls are not strong enough to handle them so that it takes a control method that can make a DC motor adapt to the environmental conditions while maintaining the performance of the system without having to re-set parameters. To improve its performance, the DC motor has to be optimized in the control system. A nonliniear control system approach for a DC motor such as a sliding mode control design as in [5], backstepping control as in [6,7], and an adaptive control as in [8-13] have been discussed. Based on paper as in [5-13], the adaptive control approach is the most suitable type of control to treat conditions such as the disturbance of a DC motor. So, we used the adaptive control based on Model Reference Adaptive
Control (MRAC) to control our DC motor model. It will be designed in Simulink to simulate the control system.

This paper presents the application of an adaptive control of for a DC motor. The uncertainties include parametric variations in the time-varying disturbance. We chose to use MRAC with the MIT rule method for the adaptation mechanism and estimate controller parameter to match the reference model. The purpose of this paper is to obtain an adaptive control design that is resistant to disturbance by using Simulink for modeling the dynamics of the system so that it can easily obtain a solution of modeling without having too many calculations of the complex mathematical equations.

This paper is organized as follows. Section II presents the DC motor description which contains the modeling dynamic system and get the transfer function for DC motor speed. Section III presents the design of the proposed MRAC. Section IV presents the result of simulation, and the last section presents the conclusions based on the purpose of the paper with future work suggestion.

\section{DC MOTOR DESCRIPTION}

A DC motor directly provides rotary motion and coupled with wheels or drums and cables, it can provide translational motion. The electric equivalent circuit of the armature and the free-body diagram of the rotor are shown in Fig. 1.

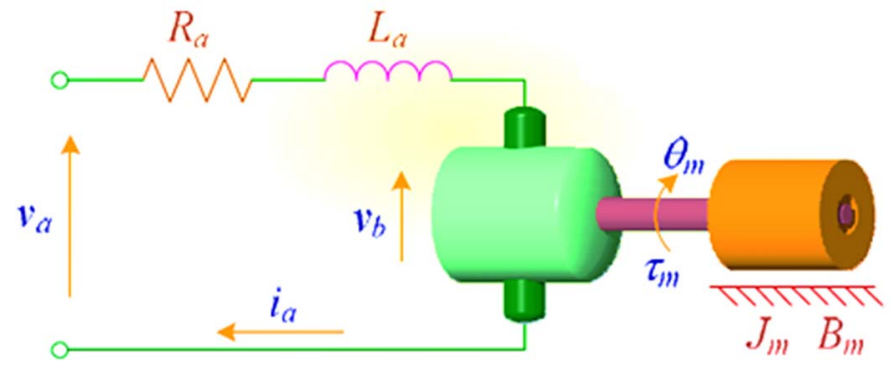

Fig.1. Schematic representation of a DC motor system [2]. 
The value of the DC motor parameters to be used in this simulation are shown in Table 1.

TABLE I. DC MOTOR PARAMETER AND VALUE [2].

\begin{tabular}{|c|c|}
\hline Parameter & Value \\
\hline Momen of inertia & $J_{m}=0,000052 \mathrm{Kg} . \mathrm{m}^{2}$ \\
\hline Friction coefficient & $B_{m}=0,01 \mathrm{~N} . \mathrm{ms}$ \\
\hline Back EMF constant & $K_{b}=0,235 \mathrm{~V} / \mathrm{rad} \mathrm{s}$ \\
\hline Torque constant & $K_{a}=0,235 \mathrm{Nm} / \mathrm{A}$ \\
\hline Electric resistance & $R_{a}=2 \mathrm{ohm}$ \\
\hline Electric inductance & $L_{a}=0.23 \mathrm{H}$ \\
\hline Input voltage & $v_{a}$ \\
\hline Back EMF voltage & $v_{b}$ \\
\hline Armature Current & $i_{a}$ \\
\hline Developed Torque & $\tau_{m}$ \\
\hline Angle of motor shaft & $\theta$ \\
\hline Load Torque & $T_{L}$ \\
\hline
\end{tabular}

Based on Fig. 1, we can derive the following governing differential equations based on Newton's $2^{\text {nd }}$ law as in (1) and Kirchhoff's voltage law as in (2) that represent the state of the dynamics of the DC motor system, so the equation as follows.

$$
\begin{gathered}
J_{m} \frac{d^{2} \theta}{d t^{2}}=\tau(t)-B_{m} \frac{d \theta}{d t} \\
L_{a} \frac{d i_{a}}{d t}=v_{a}(t)-R_{a} i_{a}(t)-v_{b}(t)
\end{gathered}
$$

Furthermore, to obtain a transfer function, the equations in (1) and (2) were changed to the frequency domain using the Laplace transform in order to obtain the transfer function in (3). Including the parameters in Table 1, we can get the transfer function of a DC motor for controlling speed.

$$
\frac{\omega(s)}{V_{a}(s)}=\frac{k_{a}}{\left[\left(L_{a} s+R_{a}\right)\left(J_{m} s+B_{m}\right)+k_{a} k_{b}\right]}
$$

The transfer function in (3) presents that the rotational speed, $\omega$ is considered the output and the armature voltage is considered the input, $V_{a}$ Then we converted into a block diagram as shown in Fig. 2.

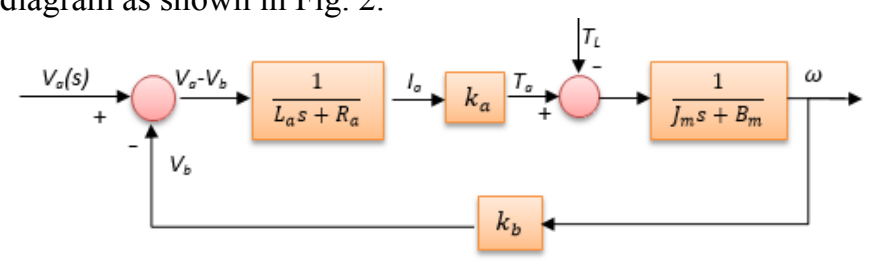

Fig.2. Block diagram of DC motor.

Fig. 2 is converted into a Simscape form in Simulink to obtain Fig. 3 that represents actual DC motors.

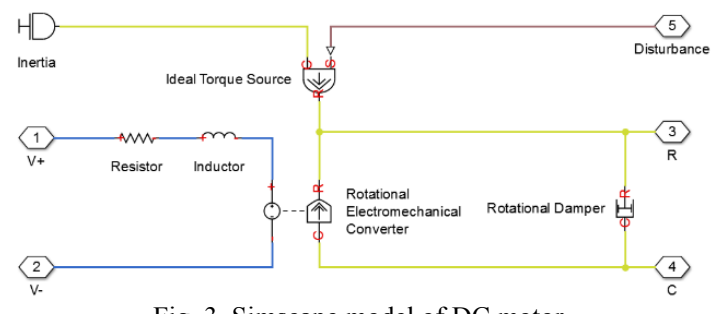

Fig. 3. Simscape model of DC motor.

\section{Design Of Proposed MRAC}

Generally, a model-reference adaptive control system can be schematically represented by Fig. 4. It is composed of four parts: a plant containing unknown parameters, a reference model for compactly specifying the desired output of the control system, a feedback control law containing adjustable parameters, and an adaptation mechanism for updating the adjustable parameters. The output of the system is compared to a desired response from a reference model. The control parameters are updated based on this error. The goal is for the parameters to converge to ideal values that cause the plant response to match the response of the reference model [14].

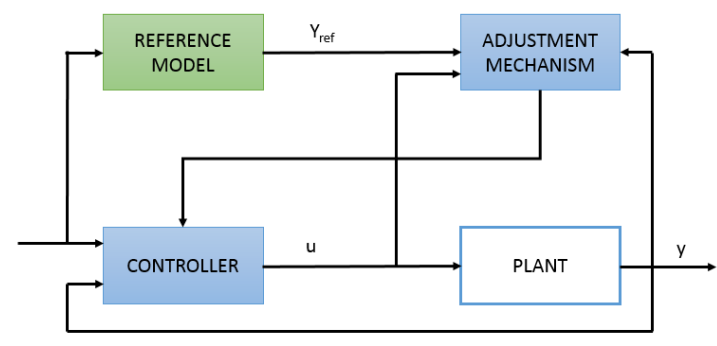

Fig. 4. Block diagram of model-reference adaptive system [15].

There are several methods which are used in the adaptation mechanism such as the MIT rule, Lyapunov theory, passivity theory etc [8]. Here the MIT rule adaptation mechanism is used to tune the controller parameter $[14,15]$ that is represented by Fig. 5.

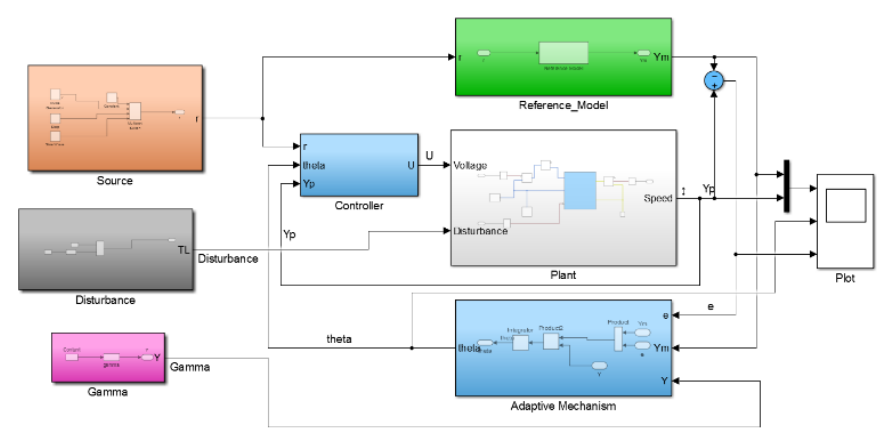

Fig. 5. Simulink block diagram of MRAC based on MIT rule.

\section{A. Reference Model}

A reference model is used to specify the ideal response of the adaptive control system to the external command. Intuitively, it provides the ideal plant response which the adaptation mechanism should seek in adjusting the parameters. A reference model can be obtained by performing linearization 
to the plant model by entering the parameters in Table 1 then obtaining the transfer function in (4) which describes the response of the DC motor speed [14,15].

$$
\frac{\omega(s)}{V_{a}(s)}=\frac{19649}{(s+162.2)(s+38.7)}
$$

We then get the unit step open-loop response plot in Fig. 6 for the system in (4).

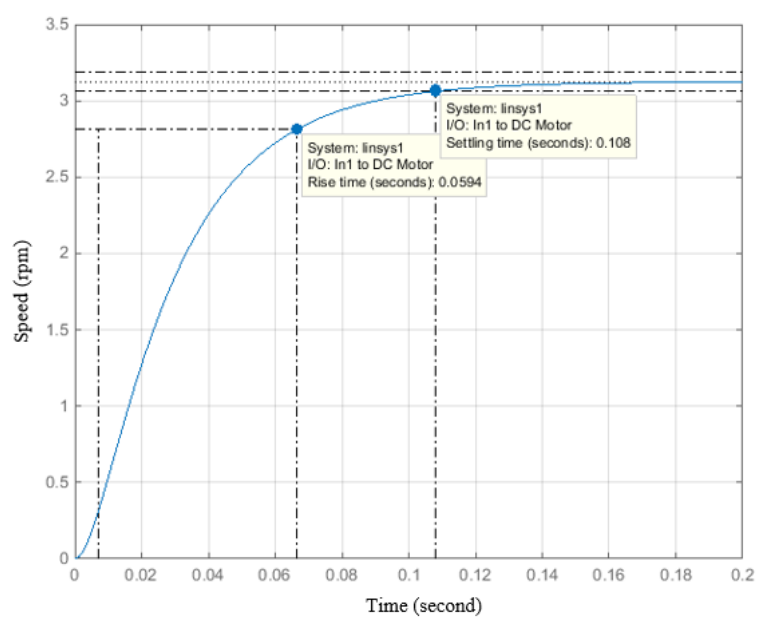

Fig. 6. Step response for open-loop system.

Based on Fig. 6, and the roots of the characteristic equation in (4), the system has a stable condition. Furthermore, to prevent the effects of the higher order frequency and have a good response, we consider closely a first-order model which approximates the original motor model. Fig. 7, presents how the first-order model is close to the original response.

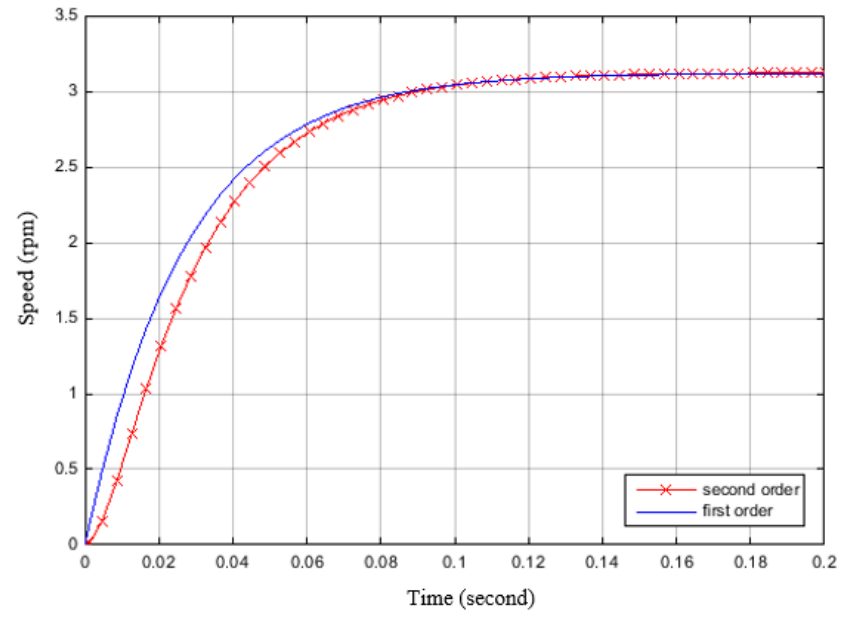

Fig.7. first-order approximation to the motor model.

By calculating the time constant and the amplitude in Fig 6, we then get the transfer function in (5) for first-order model.

$$
\frac{\omega(s)}{V_{a}(s)}=\frac{3.12}{0.027 s+1}
$$

The first-order model in (5) is to be controlled by tuning the PID to get the desired response as a reference model. Fig. 8 is the result of tuning the PID with Table 2 and describes the value of the PID gain and the desired response system.

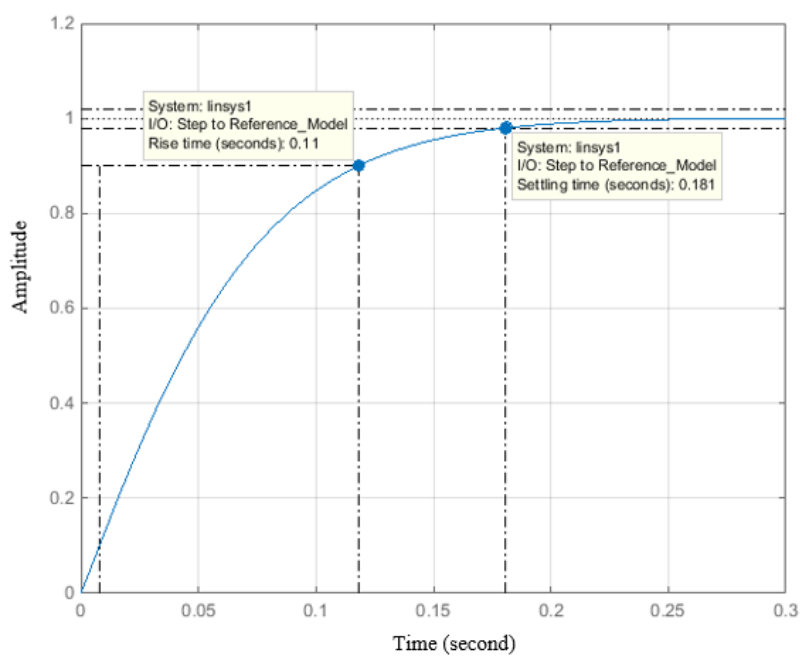

Fig.8. Tuning PID response for closely first-order system.

TABLE 2 . VALUE OF PID GAIN AND THE DESIRED RESPONSE SYSTEM OF REFERENCE MODEL.

\begin{tabular}{|c|c|c|}
\hline \multirow{3}{*}{ Controller } & $\mathrm{P}$ & Tuned \\
\hline \multirow{4}{*}{ Parameters } & $\mathrm{I}$ & 0.10997 \\
\cline { 2 - 3 } & $\mathrm{D}$ & 5.837 \\
\cline { 2 - 3 } & $\mathrm{N}$ & 0 \\
\hline \multirow{3}{*}{ Performance } & Rise time & 0.11 seconds \\
\cline { 2 - 3 } & Settling time & 0.181 seconds \\
\cline { 2 - 3 } & Overshoot & $0 \%$ \\
\hline
\end{tabular}

Finally, we get the linearization of the PID closed loop control for the first-order model. It is described as a transfer function in (6).

$$
\frac{\omega(s)}{V_{a}(s)}=\frac{12.71 s+674.5}{s^{2}+49.7 s+674.5}
$$

\section{B. Plant}

The plant is assumed to have a known structure. We modeled the DC motor plant by using simscape in Fig. 9. It describes the actual DC motor without modeling the mathematic equation.

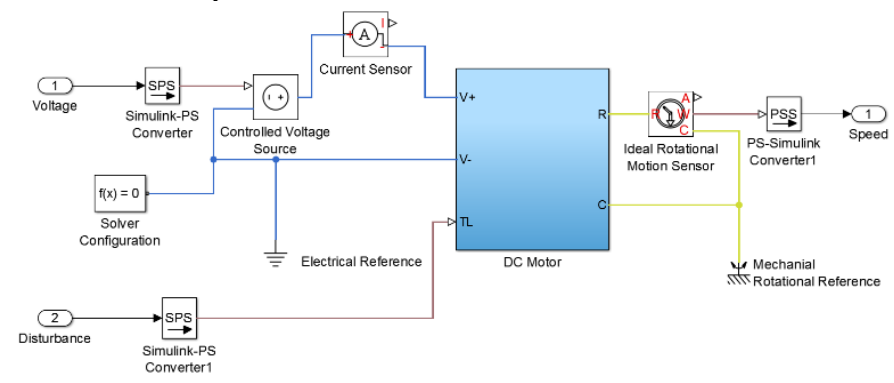

Fig.9. Simscape model for DC motor. 


\section{Adaptation Mechanism}

The adaptation mechanism is used to adjust the parameters in the control law. In MRAC systems, the adaptation law searches for parameters such that the response of the plant under adaptive control becomes the same as that of the reference model. The objective of the adaptation is to make the tracking error converge to zero [14]. We used the MIT rule to adapt the controller [15].

The MRAC begins by defining the tracking error (e) in (7). As shown in Fig. 5. $Y_{p}$ is the plant output and $Y_{m}$ is the reference model output:

$$
e=Y_{p}-Y_{m}
$$

To present the MIT rule, we will consider a closed-loop system in which the controller has one adjustable parameter theta $(\theta)$. (8) presents equation of the error cost function of theta with $\mathrm{J}$ is given as a function of theta.

$$
J(\theta)=\frac{1}{2} e^{2}
$$

The goal is to minimize to zero. For this reason, the change in the parameter theta in the direction of the negative gradient of J. So the equation becomes (9) with $\gamma$ is an adaptation gain.

$$
\frac{d \theta}{d t}=-\gamma \frac{\partial J}{\partial \theta}=-\gamma e \frac{\partial e}{\partial \theta}
$$

We will now use the MIT rule to obtain a method for adjusting the parameter theta as shown in Fig. 10. Consider the problem, Fig. 6, presents adjusting a feed-forward gain. In this problem, it is assumed that the process is a liniear plant which is built by the model Simscape, $G(s)$, the reference model, $G_{m}(s)$ and the control law, $u$.

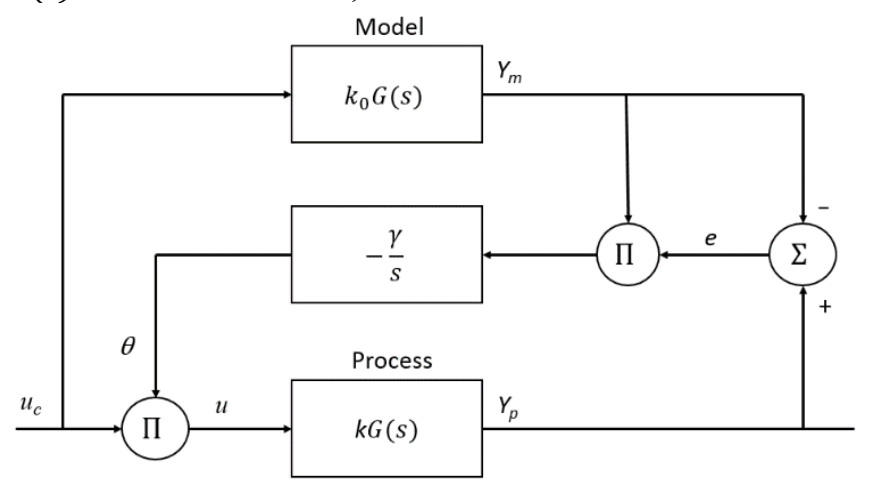

Fig. 10. Block diagram of MRAC for adjustment of a feedforward gain based on the MIT rule.

Control law $u$, described in (10) is composed of a control signal $u_{c}$, and theta $\theta$, described in (11). We use the PID control to generate a control signal. The type of method used to obtain the PID control gain is to use the PID tuning block in Simulink.

$$
u=\theta u_{c}
$$

$$
\theta=\frac{k_{0}}{k}
$$

Based on Fig. 10, we can generate the error in (12) which is $p$ $=d / d t$ as the differetial operator and arrive at the sensitivity derivative in (13).

$$
\begin{gathered}
e=Y_{p}-Y_{m}=k G(p) \theta u_{c}-k_{0} G(p) u_{c} \\
\frac{\partial e}{\partial \theta}=k G(p) \theta u_{c}=\frac{k_{0}}{k} Y_{m}
\end{gathered}
$$

Based on the equation that were described in (9) and (13), we can obtain the MIT rule for adaptation law in (14).

$$
\frac{d \theta}{d t}=-\gamma^{\prime} \frac{k}{k_{0}} Y_{m} e=-\gamma Y_{m} e
$$

\section{Controller}

The controller that is described in Fig. 11 is usually parameterized by a number of adjustable parameters. The controller should have a perfect tracking capacity in order to allow the possibility of tracking convergence. For this simulation, we use the PID controller to set the response system of the plant and get the PID gain. Fig. 12 and Table 3 show the undesirable design of the plant with a high overshoot. The purpose of the design is a differentiator between the plant design with the design of the reference model so the adaptation process towards a zero error value with changes in theta will be seen clearly.

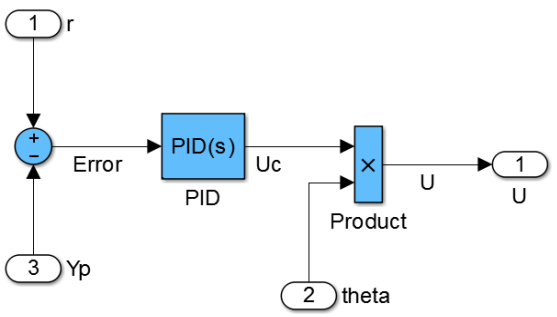

Fig. 11. Control law with PID controller.

By using tuning the PID block in Fig. 11, we can get the values of the gain with a response like those shown in Table 2 and Fig. 8. 


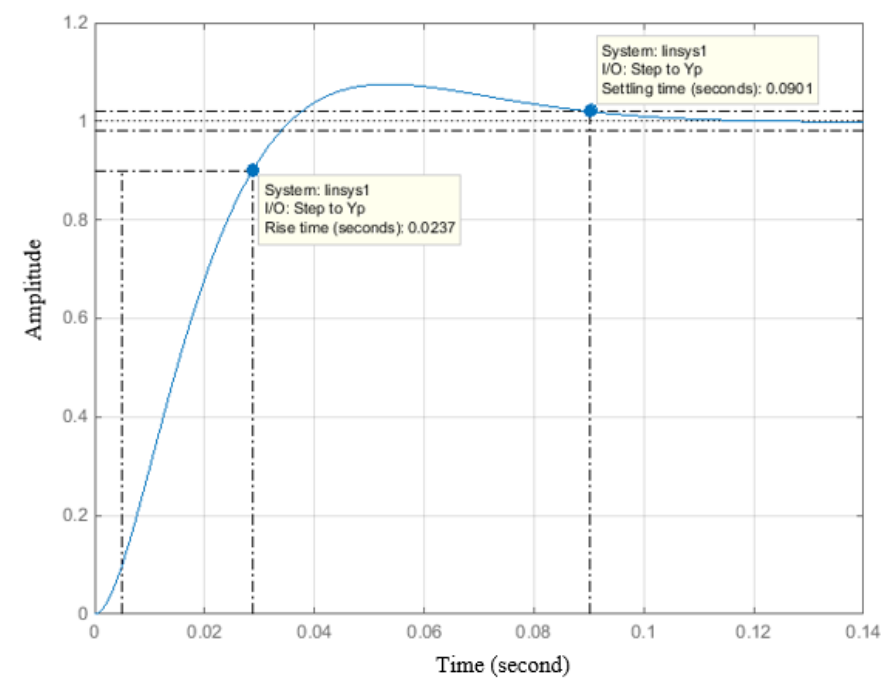

Fig.12. Tuning PID response for plant.

TABLE 3 . VALUE OF PID GAIN AND THE UNDESIRED RESPONSE SYSTEM OF PLANT.

\begin{tabular}{|c|c|c|}
\hline \multirow{3}{*}{ Parameters } & Tuned \\
\hline \multirow{4}{*}{ Controller } & $\mathrm{P}$ & 0.39442 \\
\cline { 2 - 3 } & $\mathrm{I}$ & 24.7002 \\
\cline { 2 - 3 } & $\mathrm{D}$ & -0.0018871 \\
\cline { 2 - 3 } & $\mathrm{N}$ & 209.0118 \\
\hline \multirow{3}{*}{ Performance } & Rise time & 0.0237 seconds \\
\cline { 2 - 3 } & Settling time & 0.0901 seconds \\
\cline { 2 - 3 } & Overshoot & $7.54 \%$ \\
\hline
\end{tabular}

\section{Simulation Results}

This section demonstrates the simulation result of the DC motor speed control. Before starting the simulation, we entered the parameter values from Table 1 into the workspace MATLAB which was used as input in the modeling of the MRAC on Simulink. These are some of the results that will be discussed in this paper. One is the relationship between the gamma value of the speed of adaptation, which is seen from the errors and generated theta.

The pulse generator in Simulink produces some square signals which are used as input to the MRAC system as shown in Fig. 13. The signal (Yp) that is produced from the plant has been successfully matched to the reference signal (Ym). It is described by the error signal in Fig. 13 towards zero and the constant signal of theta as shown in Fig. 14. Furthermore, the changes of square theta signal which is produced by adaptation mechanism are shown in Fig. 15. Another result is that when given a disturbance torque (TL) on the system as shown in Fig. 16 the plant signal also has been asymptotically stable to the reference signal. It is described by the error signal in Fig. 17 that goes to zero and the constant signal of theta as shown in Fig. 18.
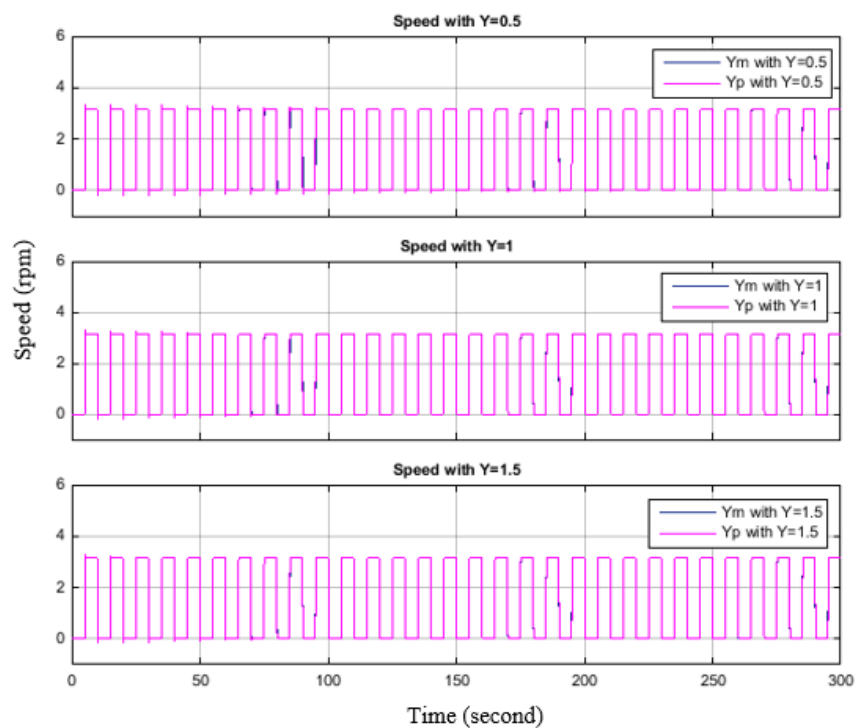

Fig. 13. Square signal as input to MRAC.
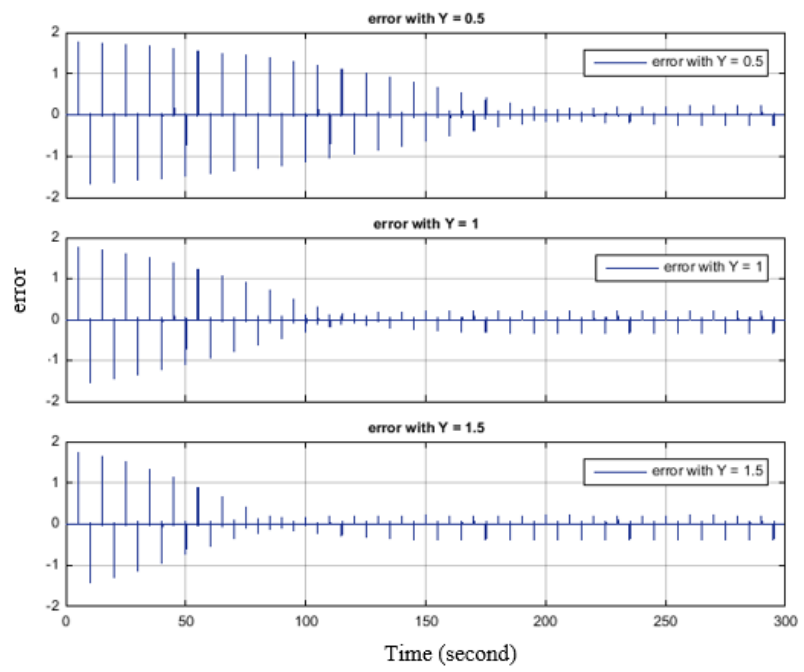

Fig. 14. Square error signal which is minimized to zero.
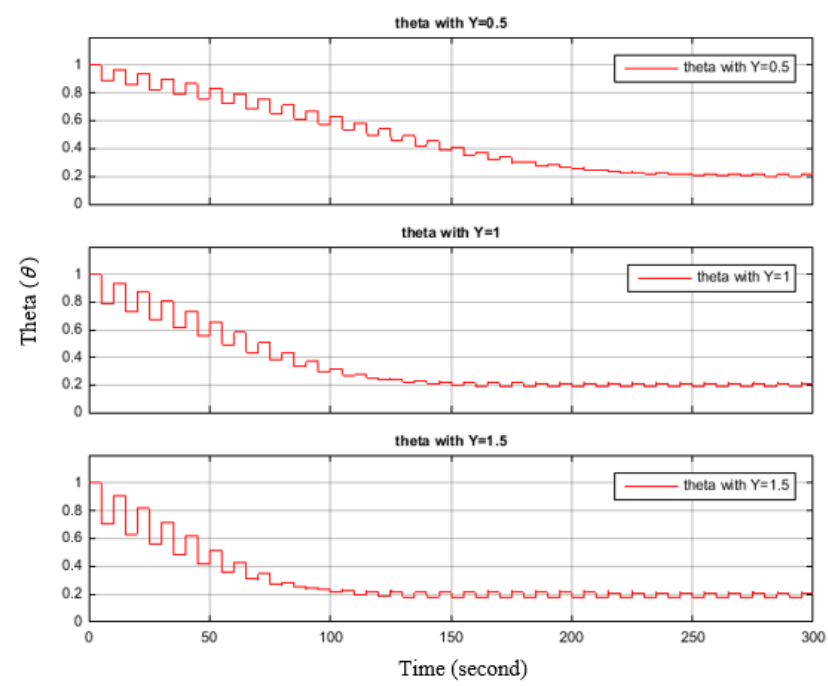

Fig. 15. The changes of square theta signal which is produced by adaptation mechanism. 

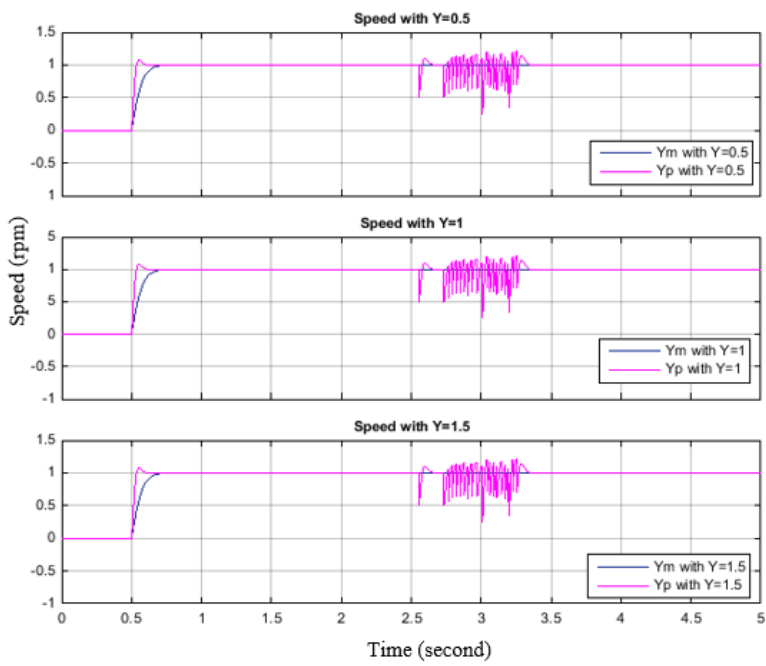

Fig. 16. Disturbance signal as input to MRAC.
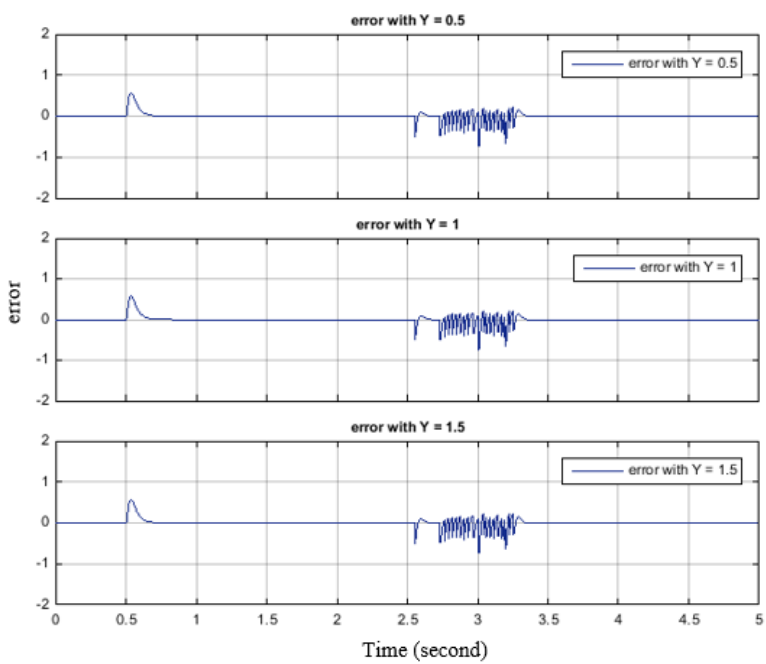

Fig. 17. Disturbance error signal which is minimized to zero.

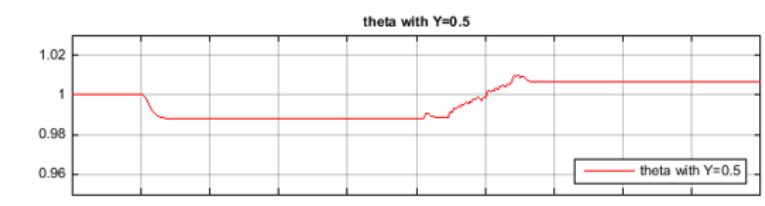

theta with $Y=1$

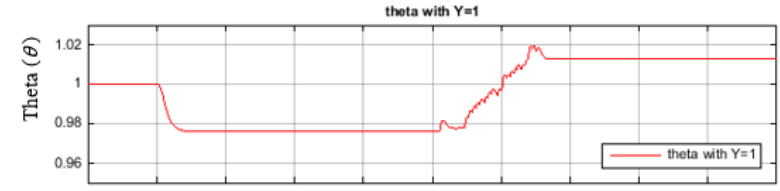

theta with $Y=1.5$

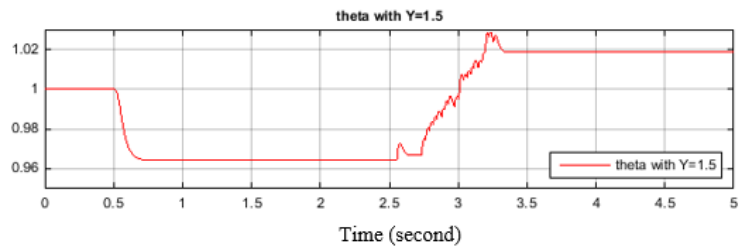

Fig. 18. The changes of disturbance theta signal which is produced by adaptation mechanism.

\section{CONCLUSION}

The simulation results showed that the MIT rule has been able to make the adaptive control system stable and resistant to varying disturbance. Increasing the value of gamma causes the system to adapt more quickly, but if the gamma value is too large, the system is very sensitive and unstable. For future work, it will be valuable to implement the MRAC design into hardware.

\section{REFERENCES}

[1] Munadi, Tomohide Naniwa, "Experimental verification of adaptive dominant type hybrid adaptive and learning controller for trajectory tracking of robot manipulators", Journal of Robotics and Mechatronics, Vol. 25. 737-747.

[2] Munadi, M. A. Akbar, "Simulation of fuzzy logic control for DC servo motor using Arduino based on MATLAB/Simulink", Intelligent Autonomous Agents, Networks and Systems (INAGENTSYS), 2014 IEEE International Conference, 2014, 42 - 46.

[3] M.J. Burridge and Z. Qu, "An improved nonlinear control design for series DC motors", Computers and Electrical Engineering 29, 2003, 273 288.

[4] D. Zhao and N. Zhang, "An Improved Nonlinear Speed Controller for Series DC Motors", Proceedings of the 17th World Congress The International Federation of Automatic Control Seoul, Korea, July 6-11, 2008

[5] A. Rhif, "Stabilizing sliding mode control design and application for a dc motor: speed control", International Journal of Instrumentation and Control Systems (IJICS) Vol.2, No.1, January 2012.

[6] A. Reza, Sahab, and Masoud, "Generalized backstepping control for dc motor", International Conference on Systems, Signal Processing and Electronics Engineering (ICSSEE'2012) December 26-27, 2012 Dubai (UAE).

[7] S.A. AL-Samarraie and Y. K. Abbas, "Design of a nonlinear speed controller for a dc motor system with unknown external torque based on backstepping approach", IJCCCE, Vol.12, No.1, 2012.

[8] R. A. Elmoudi, M. R. Abuzeid, and N. E. Shtawa, "Speed control of DC motor based on model reference adaptive controller", Al-Azhar University Engineering Journal, JAUES, Vol. 2, No. 5, 2007.

[9] Sar, S. K., \& Dewan, L., "MRAC Based PI Controller for Speed Control of D.C. Motor Using Lab View." WSEAS TRANSACTIONS on SYSTEMS and CONTROL, Volume 9, 2014.

[10] C. Khajorntraidet and J. Srisertpol, "Torque control for dc servo motor using adaptive load torque compensation", Selected topics in system science and simulation in engineering, ISBN: 978-960-474-230-1.

[11] S.E. Oltean and A.V. Duka,"Design of local model reference adaptive control for a single robotic link driven by a DC motor", The $5^{\text {th }}$ Edition of the Interdisciplinarity in Engineering International Conference "Petru Maior" University of Tîrgu Mureş, Romania, 2011.

[12] Md. A. Rahman, S. M. Ali, "Adaptive control of angular position \& angular velocity for a DC motor with full state measureable", International Journal of Engineering Research andApplications (IJERA) ISSN: 2248-9622, Vol. 3, Issue 4, Jul-Aug 2013, pp.1782-1791.

[13] G.Q. Wu, S.N. Wu, Y.G. Bai, and L. Liu, "Experimental studies on model reference adaptive control with integral action employing a rotary encoder and tachometer sensors", Sensors, 2013, 4742-4759.

[14] J.-J. E. Slotine \& W. Li, “Applied nonlinear control”, New Jersey: Prentice Hall, 1991.

[15] K.J. Astrom, and B. Wittenmark, "Adaptive control; 2nd Edition", Prentice-Hall ,1994. 